\title{
27 Rechtsordnungen oder mehr?
}

\section{Die Regelungsvielfalt des Privatrechts in der Europäischen Union}

Sebastian A. E. Martens*
A. Einleitung
B. Die Idee einer nationalen Privatrechts- ordnung ........................... 433
C. Was ist eine Privatrechtsordnung?..... 435
D. Abweichungen von der Formel: Ein
Staat $=$ eine (eigene) Privatrechts- norm.
I. Regelungsvielfalt innerhalb der Mit-
gliedstaaten ........................
1 . Besondere gesetzliche Regelun-
gen in Regionen ................ 437
a) Eigenständige regionale Gesetzgebung.............. 437
b) Zentralgesetzliche Normie- rung regionaler Besonderhei- ten.... 440

2. Gewohnheitsrecht............. 441

3. Regionale Geschäftspraxis ...... 442

4. Richterliche Rechtsfortbildung . . 442

5. Sprachbedingte Besonderheiten ........................... 443

II. Regelungseinheit über die mitgliedstaatlichen Grenzen.................. 444

1. Internationale Abkommen....... 444

2. Kooperation der Gesetzgeber.... 445

3. Rechtssetzung der Europäischen Union ......................... 446

4. Spontane Rechtsangleichung durch Wissenschaft und Praxis. . 449

E. Ergebnis 451

\section{A. Einleitung}

Die Vereinheitlichung des materiellen Privatrechts in Europa ist in den letzten Jahren zu einem immer wichtigeren Thema geworden. Der gegenwärtige Zustand einer verwirrenden Vielfalt von Privatrechtsnormen in den Mitgliedstaaten der Europäischen Union wird von vielen als unbefriedigend empfunden und nicht nur von der Kommission als Hindernis für einen funktionierenden gemeinsamen Binnenmarkt angesehen. Als eine mögliche Lösung dieser Probleme wurde ein 28. europäisches Privatrechtsregime vorgeschlagen, ${ }^{1}$ das von den Bürgern frei wählbar neben die nationalen Rechtsordnungen treten sollte. Auf diese Weise sollte das reiche rechtskulturelle Erbe Europas erhalten bleiben und doch zugleich ein optionaler einheitlicher Regelungsrahmen für die ganze Union geschaffen werden.

Der Idee einer 28. europäischen Rechtsordnung liegt die Vorstellung zugrunde, daß derzeit in der Europäischen Union 27 Privatrechtsordnungen existierten, entsprechend den 27 Mitgliedstaaten. Demgegenüber wurde von Rechtswissenschaftlern bereits darauf hingewiesen, daß zumindest Schottland ein eigenes Privatrechtssystem besitzt, ${ }^{2}$ und in Spanien gibt es ebenfalls eine alt-ehrwürdige Tradition regional un-

* Dr. habil. Sebastian A. E. Martens, M.Jur. (Oxon.), ist Referent am Max-Planck-Institut für ausländisches und internationales Privatrecht in Hamburg und vertritt derzeit eine Professur für Bürgerliches Recht und Römisches Recht an der Universität Passau.

1 Vgl. nur Reding, Rede v. 14.5.2010 (zu finden unter: http://ec.europa.eu/commission_2010-2014/reding/multimedia/speeches/index_de.htm); Tamm, GPR 2010, 281 ff.; skeptischer Leutheusser-Schnarrenberger, ZEuP 2011, 451, $45 \overline{5}$ f.;.

2 Vgl. nur etwa Smits, in: Örücü/Nelken (Hrsg.), Comparative Law - A Handbook, 2007, S. $219,220$. 
terschiedlicher Regelungen im Privatrecht. ${ }^{3}$ Zudem lassen sich die Privatrechtsordnungen auch im übrigen Europa nicht immer streng anhand der mitgliedsstaatlichen Grenzen voneinander scheiden. Vielmehr gibt es überall in Europa privatrechtliche Regelungen ganz unterschiedlicher regionaler Reichweite. Die Formel „Ein Mitgliedstaat $=$ Eine Privatrechtsordnung " basiert auf dem historisch-kontingenten Ideal eines national einheitlichen Privatrechts (dazu unter B.). Sie setzt dabei einen kaum geklärten Begriff der Privatrechtsordnung voraus (dazu unter C.). Die komplexe Regelungslage im europäischen Privatrecht kann indes nicht auf eine einfache Formel gebracht werden, sondern es lassen sich nur die Gründe für die bestehende Regelungsvielfalt systematisieren (dazu unter D.).

\section{B. Die Idee einer nationalen Privatrechtsordnung}

„Ein Volk Ein Reich Ein Recht!“ titelte die Sonderbeilage der Deutschen JuristenZeitung zum Inkrafttreten des Bürgerlichen Gesetzbuchs für das Deutsche Reich am 1.1.1900. ${ }^{4}$ Die hier stolz proklamierte Einheit von Nation, Staatsgebiet und (Privat-)Recht ist freilich eine verhältnismäßig junge Idee, deren Geburt in engem $\mathrm{Zu}$ sammenhang mit den Vorstellungen der Aufklärung und dem Entstehen der modernen Nationalstaaten in Europa steht. ${ }^{5}$ So hatte das römische Recht in der Antike keineswegs in jedem Rechtsstreit innerhalb des römischen Reichs Anwendung gefunden, sondern war in seiner Geltung auf die Beziehungen zwischen römischen Bürgern beschränkt. ${ }^{6}$ Unterworfenen Städten wurde regelmäßig gestattet, weiter nach ihrem eigenen Recht zu leben, so daß es eine große Vielfalt lokal unterschiedlicher Regelungen gab. Allerdings erkannte man schon damals, daß ein gewisser Regelungskern des Privatrechts offenbar grundsätzlich allen Völkern gemeinsam war. $^{7}$

Diese augenscheinlich kulturell und zeitübergreifende Gültigkeit bestimmter privatrechtlicher Normen ermöglichte es den mittelalterlichen Juristen nach der Wiederentdeckung der römischen Digesten, deren Autorität als ratio scripta zu postulieren $^{8}$ und diese Autorität sogar in weitem Umfang gegenüber den lokalen Rechten durchzusetzen. Zu einer vollständigen Verdrängung der lokalen Rechte durch das rezipierte römische Recht kam es jedoch nie, so daß die Einheit des sogenannten ius

3 Vgl. einführend Becker, ZEuP 1996, 88 ff.

4 Der Kupferstich des Titels ist abgedruckt bei Schulte-Nölke, Das Reichsjustizamt und die Entstehung des Bürgerlichen Gesetzbuchs, 1995, Abb. 8.

5 Vgl. auch schon Wieacker, in: ders., Industriegesellschaft und Privatrechtsordnung, 1974, S. 79 ff.

6 Vgl. Honsell/Mayer-Maly/Selb, Römisches Recht, 4. Aufl. 1987, \$26 I, S. 52 mwN.

7 Vgl. nur Gaius, Inst. I,1.

8 Der Ausdruck „ratio scripta“ findet sich im hier verwendeten Sinne allerdings weder bei den Glossatoren, noch bei den Kommentatoren, sondern hat seinen Ursprung erst in Frankreich im frühen 17. Jahrhundert, vgl. grundlegend Guzmán, Ratio scripta, 1981, S. 10 ff., 80 ff. Den frühen mittelalterlichen Juristen schien die allgemeine Geltung des römischen Rechts noch durchaus unproblematisch, das in seiner intellektuellen Qualität den zeitgenössischen Statuten weit überlegen war. 
commune vor allem eine intellektuelle Einheit der Rechtswissenschaft war, die von ihr, jeweils abhängig von ihrer (Macht-)Stellung, in die Praxis vermittelt wurde.

Erst in der Aufklärung wurde die Vorstellung einer tradierten ratio scripta, dann freilich grundstürzend, erschüttert. Geltung schien nunmehr allein das positive, d.h. das vom souveränen Herrscher gesetzte Recht für sich beanspruchen zu können. Das Recht war damit als Herrschaftsmittel entdeckt worden, mit dessen Hilfe sich die Gesellschaft nach Belieben gestalten ließ. ${ }^{9}$ Die seit dem Ende des 18. Jahrhunderts entstandenen Zivilrechtskodifikationen sollten daher auch dem politischen Zweck dienen, die jungen Nationalstaaten zu einen und ihnen jeweils eine gemeinschaftstützende Grundlage zu geben. ${ }^{10}$ Diese Kodifikationen waren gewissermaßen „, bürgerliche Verfassungen“ und damit zumindest zum Teil Ersatz für volle politische Verfassungen, die sich (noch) nicht durchsetzen ließen. ${ }^{11}$ So läßt sich auch erklären, daß ausgerechnet Zivilrechtskodifikationen in vielen europäischen Ländern zu Symbolen der nationalen Identifikation werden konnten, obwohl die inhaltlichen Übereinstimmungen unter ihnen überwiegen und selbst ihre Unterschiede regelmäßig nicht auf kulturellen Besonderheiten, sondern zumeist auf historischen Zufälligkeiten und juristisch-technischen Gründen beruhen. ${ }^{12}$

Anders als auf dem Kontinent weist die Geschichte im Vereinigten Königreich nicht nur auf dem Gebiet des Privatrechts eine größere Kontinuität auf. Das Staatswesen und auch die Rechtsprechung waren hier bereits im frühen Mittelalter vergleichsweise stark zentralisiert und wurden auf dem Weg zu den heute gültigen Strukturen (jedenfalls nach dem englischen Selbstverständnis) lediglich angepaßt, ohne daß es je zu einem radikalen Systemwechsel gekommen wäre. Das englische Common law hat seinen Ursprung daher nach traditioneller Ansicht in unvordenklicher Zeit („,time immemorial“) und ist tief mit dem Gedanken englischer Souveränität und Unabhängigkeit verbunden. ${ }^{13}$ Diese Verbundenheit schließt aber eine Offenheit für fremde Einflüsse nicht aus, und das Common law wird von England nicht als exklusives nationales Erbe beansprucht. Dies drückt sich vor allem darin aus, daß alle Rechtsordnungen der weltweiten Familie des Common law als verwandt angesehen werden, so daß nicht selten auch den Urteilen der Gerichte fremder Nationen eine gewisse

9 Vgl. vor allem Hobbes, Leviathan, 1651.

10 Vgl. zur mythenbildenden Funktion der Kodifikation Kroppenberg, JZ 2008, 905 ff., die das BGB als Beitrag zum „nation building“ ansieht (a.a.O., 912).

11 Insofern ist der Gegensatz zwischen einer politischen Gesetzgebung des frühen und mittleren 18. Jahrhunderts und den vermeintlich unpolitischen, freiheitssichernden Zivilrechtskodifikationen der Folgezeit, wie ihn Caroni, Gesetz und Gesetzbuch, 2003, S. 95 ff. konstruiert, überzeichnet. Siehe auch die grundsätzliche Kritik an einer solchen Unterscheidung bei Haferkamp, RG 19 (2011), $107 \mathrm{ff}$.

12 Vgl. etwa für die testamentarischen Formvorschriften, bei denen der Einfluß kultureller Besonderheiten doch eigentlich recht nahe läge, die umfassende rechtsvergleichende Analyse bei Reid/de Waall Zimmermann, in: dies. (Hrsg.), Testamentary Formalities, 2011, S. 432, $470 \mathrm{f.}$

13 Vgl. schon Blackstone, Commentaries on the Laws of England, Bd. 1, 1765, S. 67. 
persuasive authority zuerkannt wird. ${ }^{14}$ Die Idee der Einheit von Nation, Staat und Recht findet sich in England also bloß in einer vernünftig-unaufgeregten Version, die Raum für praktisch notwendige Relativierungen läßt.

\section{Was ist eine Privatrechtsordnung?}

Das Privatrecht steht auf dem europäischen Kontinent traditionell in einem begrifflichen Gegensatz zum Öffentlichen Recht. Die Unterscheidung zwischen diesen beiden Rechtsmassen ist nicht nur aus Sicht des deutschen Rechts von elementarer Bedeutung. Sie findet sich scheinbar bereits in einem Fragment des römischen Rechts, in dem sich der berühmte klassische Jurist Ulpian an einer Definition der beiden Rechtsgebiete zu versuchen scheint: „publicum ius est quod ad statum rei romanae spectat, privatum quod ad singulorum utilitatem "15. Mit dieser noch heute als Interessentheorie bekannten Formel wollte Ulpian freilich keineswegs die Einheit des Rechts leugnen, sondern lediglich zwei Studiengegenstände grob voneinander abgrenzen. ${ }^{16}$ Dem römischen Recht war eine strenge Unterscheidung zwischen öffentlichem Recht und Privatrecht nämlich fremd ${ }^{17}$. Zwar wurde Ulpians Formel in der frühen Neuzeit rezipiert, und man knüpfte auch im 16. und 17. Jahrhundert durchaus noch an sie an, als das öffentliche Recht sich langsam zu einem eigenständigen Rechtsgebiet entwickelte ${ }^{18}$. Doch erwies sich die Interessentheorie als untauglich sowohl für eine trennscharfe wissenschaftliche Unterscheidung als auch für die Bedürfnisse der juristischen Praxis. Dies liegt vor allem daran, dass der Gesetzgeber letztlich mit jeder von ihm erlassenen Norm (auch) öffentliche Zwecke verfolgt, so daß nach Ulpians Formel jede Norm als öffentlich-rechtlich angesehen werden könnte.

Trotz eines gewaltigen dogmatischen Aufwands und der Entwicklung zahlreicher Theorien ist eine präzise begriffliche Trennung zwischen dem öffentlichen Recht und dem Privatrecht bis heute nicht recht gelungen, und es wird sogar an dem Sinn der Unterscheidung gezweifelt. ${ }^{19} \mathrm{Da}$ die beiden Rechtsgebiete nicht zwingend voneinander unterschieden werden müssen, zeigt das Beispiel des englischen Common law, das über lange Zeit ohne eine solche Differenzierung ausgekommen ist. ${ }^{20}$ Und selbst im deutschen Recht markiert die Trennung weniger fundamentale inhaltliche Un-

14 Vgl. nur Cross/Harris, Precedent in English Law, 4. Aufl. 1991, S. 22 ff.

15 Dig. 1.1.1.2 (Ulpianus 1 inst).

16 Vgl. bereits Kaser, SDHI 17 (1951), 267, $277 \mathrm{f}$.

17 Vgl. bereits Bullinger, Öffentliches Recht und Privatrecht, 1968, S. 13 ff.

18 Vgl. Stolleis, Geschichte des öffentlichen Rechts in Deutschland, Bd. I, 1988, S. 75 ff.

19 Vgl. die ausführliche Darstellung des Streitstandes bei Schoch/Schmidt-Aßmann/Pietzner (Hrsg.), VwGO, 22. Ergänzungslieferung 2011, $\$ 40$, Rdn. 220 ff.; siehe auch Bydlinksi, AcP 194 (1994), 319, 340 ff.; Leisner, JZ 2006, 869 ff. Zu den historischen Hintergründen der Unterscheidung siehe etwa Jansen/Michaels, RabelsZ 71 (2007), 345, $388 \mathrm{ff}$.

20 Zur jüngeren Entwicklung, in der sich die Unterscheidung auch in England herausbildet, vgl. Freedland, in: ders./Auby (Hrsg.), The Public Law/Private Law Divide, 2006, S. 93 ff. 
terschiede, sondern dient vor allem der Abgrenzung der Gerichtsbarkeiten und sollte deshalb im Hinblick auf diese Funktion in Zweifelsfällen präzisiert werden. ${ }^{21}$

Ein europaweit anerkannter, exakter Begriff des Privatrechts und damit auch der Privatrechtsordnung existiert bislang nicht. Die großen kontinentalen Kodifikationen des Privatrechts orientierten sich in der Auswahl ihrer Regelungsgegenstände vor allem an den Institutionen Justinians, ${ }^{22}$ deren Materie eher nach didaktischen Gesichtspunkten als nach rechtstheoretischen oder -systematischen Kriterien bestimmt worden war. Damit blieben von Beginn an wichtige Gebiete des Privatrechts außen vor, und im Laufe der Zeit sind mehr und mehr Sondergesetze hinzugetreten, so daß in den überkommenen Kodifikationen heute nur noch ein Kern des Privatrechts seine Regelungsgrundlage findet. Nicht selten wird der Begriff der Privatrechtsordnung ohne nähere Begründung auf diesen Regelungskern beschränkt. Damit werden aber viele wichtige Materien, insbesondere des heute so bedeutsamen Wirtschaftsrechts, ungerechtfertigt ausgeschlossen. ${ }^{23}$ Die vorliegende Untersuchung soll ohne eine solche Verengung des Blickes auskommen. Es soll vielmehr allgemein untersucht werden, wo es aus welchen Gründen in Europa zu Abweichungen von der Formel „Ein Mitgliedstaat $=$ eine (eigene) Privatrechtsnorm “ kommt. Eine präzise Definition des Privatrechts ist dazu nicht nötig, sondern es genügt, dem Privatrecht solche Rechtssätze zuzuordnen, die jedermann berechtigen oder verpflichten können. Dieser Begriff des Privatrechts dürfte, bei allen Differenzen in Einzelfragen, einen grundsätzlich allgemein akzeptablen Begriffskern beschreiben. Ausgeschieden aus dem Blickfeld dieser Untersuchung sind damit Normen, bei denen mindestens ein Zuordnungssubjekt Träger von Staatsgewalt als solcher ist und die sich damit auf den Staat als solchen beziehen. ${ }^{24}$

\section{Abweichungen von der Formel: Ein Staat = eine (eigene) Privatrechtsnorm}

Die Formel „Ein Staat = eine (eigene) Privatrechtsnorm“ kann die praktisch geltende Regelungsvielfalt im europäischen Privatrecht nur sehr ungenau beschreiben. Tatsächlich gibt es zum einen innerhalb der Mitgliedstaaten der Europäischen Union

21 Vgl. bereits Bullinger, in: FS Rittner, 1991, 69, 89. Ähnliches gilt auch für das Recht der Europäischen Union, wo die Unterscheidung im Verfahrensrecht eine wichtige Rolle spielt, vgl. Arnold, ZEuP 2012, $315,317 \mathrm{ff}$.

22 Vgl. Behrends, in: ders./Sellert (Hrsg.), Der Kodifikationsgedanke und das Modell des Bürgerlichen Gesetzbuches (BGB), 2000, S. 9, 10 f. Interessanterweise wird das Privatrecht im Scotland Act 1998, der dem schottischen Parlament weite Gesetzgebungskompetenzen im Privatrecht einräumt, ebenfalls anhand des Institutionenschemas definiert, siehe s. 126 (4).

23 Interessant ist in diesem Zusammenhang, daß in England, das traditionell der Idee einer Kodifikation des Zivilrechts kritisch gegenübersteht, 2006 mit dem Companies Act 2006 gerade eine umfangreiche Kodifikation einer Kernmaterie des Wirtschaftsrechts gelungen ist, dazu umfassend der Kommentar von Schall (Hrsg.), Companies Act 2006, im Erscheinen.

24 Dies entspricht der wohl herrschenden sogenannten (materiellen) Subjektstheorie in Deutschland, vgl. Schoch/Schmidt-Aßmann/Pietzner (Hrsg.) (Fn.19), \$40, Rdn. 225 ff. Kritik am tautologischen Charakter dieser Theorie etwa bei Leisner, JZ 2006, 869, $870 \mathrm{ff}$. 
zahlreiche Sonderregelungen von lokal begrenzter Reichweite (dazu unter 1.). Zum anderen gelten manche Rechtssätze auch einheitlich über die Grenzen mehrerer Mitgliedstaaten hinweg (dazu unter 2.).

\section{Regelungsvielfalt innerhalb der Mitgliedstaaten}

\section{Besondere gesetzliche Regelungen in Regionen}

Die Mitgliedstaaten der Europäischen Union sind keine monolithischen Blöcke, sondern sie sind vielfach gegliedert und lassen, in freilich sehr unterschiedlichem Maße, regionale Besonderheiten zu. Dies gilt auch für den Bereich der Gesetze. Einige Regionen besitzen sogar eine verschieden weitreichende gesetzgeberische Autonomie im Bereich des Privatrechts und haben hier eigene Normen erlassen (dazu unter i)). In anderen Mitgliedstaaten gibt es zentralstaatliche Regelungen im Privatrecht, die nur für bestimmte Regionen gelten (dazu unter ii)).

\section{a) Eigenständige regionale Gesetzgebung}

Die Idee des Nationalstaats war und ist nur bedingt geeignet, die Autorität der Staaten in Europa zu legitimieren. Denn dies kann nur insoweit gelingen, als alle Staatsbürger davon überzeugt werden können, Mitglieder einer gemeinsamen Nation zu sein. Wo aber innerhalb eines Staates verschiedene Nationen konstruiert werden, bzw. Nationen auf Regionen verteilt sind, die sich nicht an den politischen Grenzen der europäischen Staaten orientieren, birgt die Idee des Nationalstaats nach wie vor große Sprengkraft und droht, die bestehenden Staatsstrukturen auseinanderzureißen. Um dieses gefährliche Potential zu entschärfen, haben einige europäische Staaten bestimmten Regionen unterschiedlich weite Autonomierechte eingeräumt. $\mathrm{Zu}$ diesen Autonomierechten zählen teilweise auch Gesetzgebungskompetenzen auf dem Gebiet des Privatrechts.

Die ältesten und zugleich auch weitreichendsten Kompetenzen dieser Art bestehen im Vereinigten Königreich für Schottland gemäß dem Act of Union von 1707. Dessen Art. XVIII sieht vor, „that no alteration be made in Laws which concern private Right, except for the evident utility of the subjects within Scotland“. ${ }^{25}$ In diesem Sinne besitzt auch das durch den Scotland Act 1998 neu gegründete schottische Parlament nunmehr weite Gesetzgebungskompetenzen im Privatrecht. ${ }^{26}$ Freilich hatten die schottischen Juristen bereits in den Jahrhunderten zuvor auf der Grundlage des alten Act of Union ihr eigenes Zivilrecht recht erfolgreich gegenüber dem Einfluß des englischen Common law bewahren können, so daß nach wie vor mit Recht von einer eigenständigen schottischen Privatrechtsordnung gesprochen werden kann.

25 Siehe auch Whitty, in: MacQueen/Vaquer/Espiau Espiau (Hrsg.), Regional Private Laws Codification in Europe, 2003, S. 60, 65.

26 Vgl. Whitty (Fn. 25), S. 60, 67. 
Eine recht große regionale Regelungsvielfalt im Privatrecht besteht auch in Spanien. Zwar existiert mit dem Codigó civil seit 1889 eine gesamtspanische Kodifikation, die die klassischen Kernbereiche des Zivilrechts abdeckt. Doch gemäß seinem Art. 13 Abs. 2 beansprucht der Codigó civil in weitem Umfang nur subsidiäre Geltung und läßt die sogenannten Foralrechte, die zum Zeitpunkt seines Erlasses beobachtet wurden, unberührt. Die spanische Verfassung von 1978 gewährte den autonomen Gemeinschaften zudem die Gesetzgebungskompetenz für das Zivilrecht, sofern und soweit damals in ihrem Gebiet Foralrecht bestand (Art. 149 Abs. 1 Nr. 8). Solche Foralrechte existierten im 19. Jahrhundert vorwiegend im Bereich des Erbund Familienrechts. Diese Materien sind deshalb bis heute in Spanien regional recht uneinheitlich geregelt. ${ }^{27}$

Im übrigen Privatrecht fanden vor Erlaß des Codigó civil in erster Linie das römische Recht des ius commune und die sogenannten Siete Partidas ${ }^{28}$ Anwendung. Besondere Regelungen im Schuldrecht bestanden in einem freilich immer noch bescheidenen Maß lediglich in Katalonien und Navarra. ${ }^{29}$ Allerdings gibt es heute in vielen autonomen Regionen Bestrebungen, weite Bereiche des Zivilrechts eigenständig zu regeln, indem man sich auf angeblich bereits existierende und nunmehr lediglich zu reformierende Foralrechte beruft. Besonders bedeutsam ist diese Tendenz in Katalonien. ${ }^{30}$ Art. 129 des katalanischen Autonomiestatuts enthält eine umfassende Gesetzgebungskompetenz für das Zivilrecht, soweit Art. 149 Abs. 1 Nr. 8 der spanischen Verfassung die Regelung bestimmter Materien nicht ausschließlich dem Zentralstaat zuweist. Damit wäre nach dem Wortlaut des Statuts auch der Erlaß katalanischer Gesetze in Bereichen möglich, in denen bislang noch kein Foralrecht bestand. Mit Urteil vom 28.6.2010 hat das spanische Verfassungsgericht Art. 129 des Autonomiestatuts nichtsdestotrotz für verfassungsgemäß erklärt, da mit dieser Norm inhaltlich keine Abweichung von Art. 149 Abs. 1 Nr. 8 der Verfassung intendiert gewesen sei. Eine Gesetzgebungskompetenz im Zivilrecht bestehe für Katalonien deshalb auch weiterhin nur für solche Materien, in denen im Zeitpunkt des Inkrafttretens der Verfassung bereits eigene Regelungen existierten. ${ }^{31}$ Das Projekt einer eigenen umfassenden Zivilrechtskodifikation für Katalonien ${ }^{32}$ entbehrt damit insoweit der verfassungsrechtlichen Basis, als Themen behandelt werden, die nicht bereits vom

27 Vgl. auch Meyer, informaciones 2002, 152, 154.

28 Dieses Gesetzeswerk aus dem Jahre 1265 ist eine Mischung aus spanischen, römischen und kanonischen Gedanken, vgl. Bucher, ZEuP 2004, 515, 520 f. mwN.

29 Vgl. Becker, ZEuP 1996, 88, 106.

$30 \mathrm{Zu}$ den historischen Hintergründen der besonderen Stellung Kataloniens im Hinblick auf das Zivilrecht vgl. Harty, Law and History Review 20 (2002), 349, $354 \mathrm{ff}$.

31 Sentencia 31/2010 v. 28.6.2010, Boletín Oficial del Estado Nr. 172 v. 16.7.2010.

32 Vgl. dazu etwa Martín-Casals, ZEuP 2004, 677 ff. 
alten Foralrecht abgedeckt wurden. ${ }^{33}$ Das sechste Buch der geplanten Kodifikation, das dem Schuldrecht gewidmet sein sollte, wird deshalb nicht ohne Veränderungen erlassen werden können. Die übrigen ersten fünf Bücher des katalanischen Código civil, die dem Verjährungsrecht und der caducitat, dem Recht der natürlichen Personen und dem Familienrecht, dem Recht der juristischen Personen, dem Erb- und dem Sachenrecht gewidmet sind, ${ }^{34}$ sind ungeachtet dessen mittlerweile durch Gesetz verabschiedet worden und bilden den stolzen Kern des katalanischen Zivilrechts.

Ähnlich weitreichende Gesetzgebungskompetenzen im Zivilrecht wie Schottland und die spanischen autonomen Gemeinschaften besitzen die anderen autonomen Regionen Europas nicht. In Italien hat etwa die Region Trentino-Südtirol, die das Gebiet der Provinzen Trient und Bozen umfaßt, gemäß Art. 8 Nr. 8 des Sonderstatuts für Trentino-Südtirol im Bereich des Zivilrechts lediglich die Befugnis, Gesetzesbestimmungen zur Ordnung der geschlossenen Höfe und der auf alten Satzungen oder Gepflogenheiten beruhenden Familiengemeinschaften zu erlassen. ${ }^{35}$ Im Übrigen gibt es in Italien gesetzliche regionale Unterschiede vor allem noch im Grundbuchrecht.

In Deutschland fällt das Bürgerliche Recht gemäß Art. 74 Abs. 1 Nr. 1 GG in die konkurrierende Gesetzgebungskompetenz des Bundes. Ebenfalls zur konkurrierenden Gesetzgebungskompetenz des Bundes zählen das Recht der Wirtschaft (Art. 74 Abs. 1 Nr. 11 GG) und das Arbeitsrecht (Art. 74 Abs. 1 Nr. 12 GG). Von seiner Gesetzgebungskompetenz im Bürgerlichen Recht hat der Bund in weiten Bereichen, insbesondere im Schuldrecht, vor allem durch die Kodifikation des BGB grundsätzlich umfassenden und abschließenden Gebrauch gemacht. ${ }^{36}$ Gemäß Art. 55 EGBGB treten die privatrechtlichen Vorschriften der Landesgesetze außer Kraft, soweit nicht im BGB oder im EGBGB ein anderes bestimmt ist. Neben einer Reihe von Vorbehalten für das Landesrecht im $\mathrm{BGB}^{37}$ selbst findet sich eine umfangreiche Liste solcher Ausnahmen vor allem in den Artt. 56 bis 152 EGBGB. Hierzu zählen hauptsächlich Materien des Erb- und Liegenschafts- sowie des Nachbarrechts, aber auch etwa das Recht zur Aneignung der einem anderen gehörenden, im Freien betroffenen Tauben (Art. 130 EGBGB) oder das Recht zur Benutzung eines Platzes in einer Kirche oder auf einem Friedhof (Art. 133 EGBGB). Zudem galten für einige zum Zeitpunkt

33 A.A. etwa Espiau-Espiau, in: MacQueen/Vaquer/Espiau Espiau (Hrsg.), Regional Private Laws Codification in Europe, 2003, S. 172, 178 ff.; siehe auch die aus katalanischer Sicht sehr positive Deutung der Entscheidung des Verfassungsgerichts bei Egea Fernàndez, Revista catalana de dret públic 2010, $340,345$.

34 Vgl. Martín-Casals, ZEuP 2004, 677, 680.

35 Siehe das auf dieser Grundlage erlassene südtiroler Höfegesetz, Landesgesetz v. 28.11.2001, Nr. 17.

36 Vgl. Otter, in: v. Mangoldt/Klein/Starck (Hrsg.), Kommentar zum Grundgesetz, 6. Aufl. 2010, Bd. II, Art. 74, Rdn. 11.

$37 \int \mathbb{S} 44,55$ a Abs. 1,79 Ab. 5 S. 3, 80 Abs. 3, 85, 88, 558 Abs. 4, 577 a Abs. 2 S. 2, 907 Abs. 1 S. 2, 919 Abs. 2, 979 Abs. 1 b S. 2, 1059 a Abs. 1 Nr. 2 S. 4, 1316 Abs. 1 Nr. 1 S. 2, 1558 Abs. 2 S. 1, 1600 Abs. 6 S. 1, 1784 Abs. 1, 1807 Abs. 2, 1888, 1908 f Abs. 3, 1808 i Abs. 1 S. 2, 2249 Abs. 5 S. 1 BGB. 
des Inkrafttretens des BGB bereits begründete Rechtsverhältnisse die landesrechtlichen Bestimmungen fort (Siehe Artt. 157 ff. EGBGB).

Die Bundesländer haben von den ihnen eingeräumten Gesetzgebungskompetenzen im Bürgerlichen Recht vor allem in ihren Ausführungsgesetzen zum BGB Gebrauch gemacht. Hier finden sich regelmäßig besondere Regelungen für das Nachbar- und Liegenschaftsrecht, sowie im Familien- und Erbrecht. Doch beispielsweise im bayerischen BGB-AG werden mit dem Bierlieferungsvertrag (Artt. 5 f.) und dem Leibgedingsvertrag (Artt. $7 \mathrm{ff}$.) auch zwei besondere Vertragstypen geregelt. Neben den Ausführungsgesetzen zum BGB gibt es noch einige landesrechtliche Spezialgesetze wie etwa das Höfegesetz des Bundeslandes Bremen. ${ }^{38}$

\section{b) Zentralgesetzliche Normierung regionaler Besonderheiten}

Höfeordnungen haben freilich auch dort nicht immer gesamtstaatliche Geltung, wo es an eigenen landesgesetzlichen Regelungen wie dem Bremer Höfegesetz fehlt. So wurde in Österreich das Tiroler Höfegesetz nach dem Zweiten Weltkrieg durch Bundesgesetz grundsätzlich wieder in Kraft gesetzt, ${ }^{39}$ und in Kärnten gilt für die Erbteilung bei der gesetzlichen Erbfolge nach einem Erblasser, der allein oder gemeinsam mit seinem Ehegatten, Elternteil oder Kind Eigentümer eines dort gelegenen Erbhofs gewesen ist, das Bundesgesetz vom 13. Dezember 1989 über die bäuerliche Erbteilung in Kärnten. ${ }^{40}$ Und auch in Deutschland hat die bundesrechtliche Höfeordnung einen regional begrenzten Anwendungsbereich, indem sie nur für Höfe im Gebiet der ehemaligen britischen Besatzungszone, d.h. der Bundesländer Hamburg, Niedersachsen, Nordrhein-Westfalen und Schleswig-Holstein gilt ( $\$ 1$ Abs. 1 HöfeO). Diese Gesetze der Zentralstaaten beschränken ihre Anwendbarkeit also ausdrücklich auf bestimmte Regionen.

Diese Regelungstechnik findet nicht nur im Höferecht. In Frankreich etwa regeln die Artt. L670-1 ff. Code de commerce ein besonderes Verbraucherinsolvenzverfahren. Diese sogenannte faillite civile gilt für natürliche Personen, die weder Kaufleute, noch Handwerker, noch Landwirte, noch Freiberufler sind und ihr domicil in den Départments Moselle, Bas-Rhin oder Haut-Rhin haben. ${ }^{41}$ Die Existenz dieses Sonderrechts für das Gebiet des ehemaligen Elsaß-Lothringen hat historische Gründe. Nach dem deutsch-französischen Krieg 1870 und der folgenden Annexion des Elsaß durch das neue Deutsche Reich wurde das vorher geltende französische Recht in vielen Bereichen durch deutsches Recht ersetzt oder es wurden eigenständige Gesetze für das

38 Bremisches Höfegesetz v. 19.7.1948 (Brem. GBl. S. 124), zuletzt geändert durch Art. 1 Abs. 23 Drittes Rechtsbereinigungsgesetz vom 24.11.2009 (Brem. GBl. S. 517). Dieses Gesetz tritt gemäß $\$ 32$ mit Ablauf des 31.12.2014 außer Kraft.

39 \4 Bundesgesetz vom 21.3.1947 (BGBl. Nr. 85/1947).

40 Kärtner Erbhöfegesetz 1990, BGBl. Nr. 658/1989, zuletzt geändert durch Art. XXVII des AußerstreitBegleitgesetzes, BGBl. 112/2003.

41 Einführend hierzu Delzant/Schütze, ZInsO 2008, $540 \mathrm{ff}$. 
Elsaß erlassen. Als 1918 das Elsaß zu Frankreich zurückkehrte, entschloß sich der französische Gesetzgeber, die lokalen Besonderheiten des Elsaß zunächst beizubehalten und nur schrittweise an das französische Recht anzugleichen. Regelungen, die gesetzgebungstechnisch oder aus anderen Gründen überlegen schienen, wurden sogar bei späteren Reformen unberührt gelassen und durch besondere Bestimmungen in den gesamtstaatlichen Kodifikationen, wie den Artt. L670-1 ff. Code de commerce, perpetuiert. Die Feststellung des geltenden Rechts für die Départments Moselle, Bas-Rhin und Haut-Rhin ist freilich mitunter recht kompliziert, da die gesamtstaatlichen Gesetze das lokale Recht nicht immer explizit außer Kraft setzen. Wichtige elsässische Sonderregelungen finden sich im Zivilrecht etwa noch für das Arbeitsrecht. ${ }^{42}$

\section{Gewohnheitsrecht}

Neben den Gesetzen ist das Gewohnheitsrecht in Europa allgemein als Rechtsquelle anerkannt. Rechtliche Geltung kann Gewohnheitsrecht nach herkömmlicher Ansicht unter zwei Voraussetzungen beanspruchen: Erstens muß eine bestimmte tatsächliche Übung von einer gewissen Dauer vorliegen (consuetudo oder repetitio), und diese Übung muß zweitens von einer gewissen Rechtsüberzeugung (opinio iuris vel necessitatis) getragen sein. ${ }^{43}$ Diese Voraussetzungen lassen sich auf der Ebene der großen Nationalstaaten heute kaum mehr erfüllen, und das Gewohnheitsrecht spielt deshalb als Rechtsquelle auch nur noch eine sehr untergeordnete Rolle. Eine Übung, die von einer bestimmten Gruppe allgemein und in Rechtsüberzeugung angewandt wird, kann nur in kleineren Einheiten entstehen. Daher wäre das Gewohnheitsrecht grundsätzlich eine besonders geeignete Rechtsquelle des lokalen Rechts.

Allerdings muß auch das regionale Gewohnheitsrecht die allgemeine Rechtsquellenhierarchie beachten. Es kann daher das gesamtstaatliche Recht nur insoweit verdrängen oder ergänzen, als der (hypothetische) regionale Gesetzgeber hierzu befugt wäre. ${ }^{44}$ Selbst wenn dies der Fall ist, muß bei der Annahme von lokalem Gewohnheitsrecht jedoch Zurückhaltung geübt werden, da es regelmäßig auch bei einer bestehenden allgemeinen Übung an der notwendigen Rechtsüberzeugung fehlt. So herrscht beispielsweise in vielen Teilen des deutschen Rheinlands die Sitte, daß die Arbeitnehmer am Rosenmontag unbezahlt freigestellt werden. Hieraus ergibt sich

42 Vgl. für das lokale Recht der Départments Moselle, Bas-Rhin und Haut-Rhin die Informationen des Institut du Droit Local Alsacien-Mosellan (http://www.idl-am.org/index.asp) und die von ihm herausgegebene Zeitschrift Revue du Droit Local.

43 Vgl. nur Bydlinski, Juristische Methodenlehre und Rechtsbegriff, 2. Aufl. 1991, S. 215.

44 Vgl. nur BGH, Beschluß v. 8.2.2010, Az. AnwZ (B) 80/09, AnwZ (B) 112/09, iuris, Rdn. 13; siehe auch BVerfGE 101, $312 \mathrm{ff}$. zur Verfassungswidrigkeit des ehemaligen $\mathbb{1} 13$ BORA, der bestimmte, daß ein Rechtsanwalt bei anwaltlicher Vertretung der Gegenseite ein Versäumnisurteil nur erwirken durfte, wenn er dies zuvor dem Gegenanwalt angekündigt hat. Diese Regelung beruhte auf einer langen Tradition unter den Rechtsanwälten, der teilweise sogar die Qualität lokalen Gewohnheitsrechts beigemessen wurde, vgl. Mennicke, MDR 1992, $221 \mathrm{ff}$. 
jedoch mangels einer Rechtsüberzeugung kein allgemein geltendes Gewohnheitsrecht. Ein Anspruch der Arbeitnehmer auf unbezahlte Freistellung kann sich deshalb allenfalls für konkrete einzelne Unternehmen nach den Grundsätzen einer sogenannten betrieblichen Übung ergeben. ${ }^{45}$

\section{Regionale Geschäftspraxis}

Gewohnheitsrecht gilt gegenüber den Rechtsunterworfenen auch ohne ihre Zustimmung. Eine Anknüpfung an eine privatautonome Entscheidung ist dagegen stets erforderlich, wenn bloß eine Bindung an lokale Geschäftspraktiken im Raume steht. ${ }^{46}$ So können sich etwa bestimmte Musterverträge in einem Gebiet als allgemein gebräuchlicher Standard herausbilden. Solche Standardverträge sind dann grundsätzlich objektiv in dem Sinne zu verstehen, wie es in dem jeweiligen Gebiet üblich ist. Rechtliche Geltung können indes auch Standardverträge selbstverständlich nur dann beanspruchen, wenn ihre Bestimmungen mit den allgemeinen, und meist überregional geltenden gesetzlichen Vorgaben im Einklang stehen.

\section{Richterliche Rechtsfortbildung}

Das praktisch durchsetzbare Recht wird nicht allein durch gesetzliche Vorschriften bestimmt, sondern auch durch die Gerichte, die die Bedeutung dieser Vorschriften im jeweiligen Verfahren erst autoritativ konkretisieren. Rechtseinheit besteht in einer bestimmten Region aus Sicht der Praxis daher nur insoweit, als die Gerichte dem Recht überall den gleichen Inhalt zusprechen. Die europäischen Rechtsordnungen versuchen mit unterschiedlichen Mitteln, eine solche einheitliche Rechtsprechung zu garantieren. ${ }^{47}$ Während in den Ländern des Common law die Gerichte an die Präjudizien übergeordneter Instanzen durch das Prinzip des stare decisis rechtlich gebunden sind, sollen auf dem Kontinent umfassende verfahrensrechtliche Vorschriften die Rechtseinheit sichern. Ein zentrales Höchstgericht dient dabei der Kontrolle der unteren Instanzen.

Inwieweit beide Systeme ihre einheitsstiftende Funktion erfüllen können, hängt entscheidend davon ab, welche kontrollfreien Spielräume den unteren Instanzen eingeräumt werden. Hier bestehen in den Rechtsordnungen Europas große, auch rechtskulturell bedingte Unterschiede, da die Vorstellungen von der Rolle und Aufgabe des Richters weiterhin stark divergieren. Eine für den außenstehenden Betrachter äußerst weitgehende Freiheit wird in Frankreich dem juge du fond zugestanden. Denn die Cour de cassation beschränkt sich in ihrer Kontrolle streng auf Rechtsfragen. ${ }^{48} \mathrm{Sie}$

45 Vgl. BAGE 73, $1 \mathrm{ff}$.

46 Vgl. etwa OLG Hamburg, MDR 1997, 810 f. zur Einbeziehung der Hamburger Lagerungsbedingungen in einen Lagervertrag nur durch Einbeziehungsvereinbarung; zustimmend Mankowski, EWiR 1997, 895 ff.

47 Vgl. dazu bereits Martens, JZ 2011, 348, 351, $354 \mathrm{ff.}$

48 Zur Kontrolle durch die Cour de cassation und des pouvoir souverain des juge du fond einführend Buffet, in: Molfessis (Hrsg.), La Cour de cassation et l'élaboration du droit, 2004, S. 113 ff. 
überläßt zudem die Anwendung ausländischen Rechts, lokaler Gewohnheitsrechte und von Verträgen grundsätzlich dem juge $d u$ fond. ${ }^{49}$ Aber auch im Übrigen kann die Cour de cassation ihre Kontrolldichte selbst festlegen, indem sie die gesetzlichen Begriffe mehr oder weniger präzise definiert und damit den kontrollfreien Bereich der Beurteilung des Sachverhalts durch den juge $d u$ fond bestimmt. ${ }^{50}$ Soweit dieser pouvoir souverain des juges $d u$ fond reicht, gibt es in Frankreich folglich keine Rechtseinheit, und die Urteile können in den Gerichtsbezirken dann sehr unterschiedlich ausfallen ${ }^{51}$.

Regionale Unterschiede in der Rechtsprechung sind allerdings kein spezifisch französisches Problem, sondern werden etwa auch in Deutschland beobachtet. Regelmäßig beklagt man hier beispielsweise im Arbeitsrecht ${ }^{52}$ oder im Zivilprozeßrecht $^{53}$ ein (vermeintliches) „Nord-Süd-Gefälle“ der instanzgerichtlichen Rechtsprechung und meint damit, daß es unterschiedliche Rechtsansichten innerhalb der verschiedenen OLG-Bezirke gibt.

\section{Sprachbedingte Besonderheiten}

Regionale Unterschiede im Privatrecht können sich schließlich aus sprachlichen Differenzen ergeben. Denn sprachliche Äußerungen haben grundsätzlich den Inhalt, der ihnen im Gebrauch der jeweiligen Sprachengemeinschaft zukommt. Solche Sprachengemeinschaften werden nicht immer durch das gesamte Staatsvolk gebildet. Vielmehr gibt es gerade in den größeren europäischen Staaten vielfältige Dialekte oder sonstige regionale Besonderheiten, die sich auch auf den Rechtsverkehr insbesondere im Vertragsrecht auswirken können. Ein bekanntes Beispiel aus der zivilrechtlichen Anfängerübung zur Frage der Auslegung von Willenserklärungen in Deutschland ist der kölsche „halve Hahn“, den ein Gast in der falschen Annahme bestellt, es handele sich um ein halbes Hähnchen, und der sich dann in Köln tatsächlich nur als ein Roggenkäsebrötchen entpuppt. ${ }^{54}$

Schwierigere Probleme für eine Rechtsordnung können bei echter Mehrsprachigkeit entstehen. Zwar läßt sich, wie das Beispiel der Schweiz zeigt, mit viel Aufwand auch hier der Rechtsdiskurs über die Sprachgrenzen hinweg grundsätzlich aufrecht erhalten, und Gesetze können mehrsprachig redigiert werden, wenn die Autoren tatsäch-

49 Vgl. Héron/Le Bars, Droit judiciaire privé, 4. Aufl. 2010, Rdn. 800 mwN.

50 Sog. appréciation souveraine des juges du fond, Héron/Le Bars (Fn. 49), Rdn. 802.

51 Vgl. etwa zu unterschiedlichen regionalen Praktiken bei der Kontrolle von Scheidungsfolgevereinbarungen in Frankreich Claux/David (Hrsg.), Droit et Pratique du Divorce, 2010, 135.252 Fn. 4.

52 Vgl. etwa Ege, BB 2010, 898 f.; Mantz, CR 2008, $52 \mathrm{ff}$.

53 Vgl. Enzensberger, ZErb 2006, $264 \mathrm{f}$.

54 Vgl. nur Boemke/Ulrici, BGB Allgemeiner Teil, 2009, S. 126; Eltzschig/Wenzel, Die Anfängerklausur im BGB, 3. Aufl. 2008, S. $71 \mathrm{ff}$; Hübner, Allgemeiner Teil des Bürgerlichen Gesetzbuches, 2. Aufl. 1996, Rdn. 749; siehe auch Larenz/Wolf, Allgemeiner Teil des Bürgerlichen Rechts, 9. Aufl. 2004, $\$ 28$ Rdn. 53 m.w.N. 
lich alle Sprachen sprechen und somit die verschiedenen Textfassungen parallel entwerfen können. ${ }^{55}$ Aber Sprachgrenzen haben dennoch stets das Potential zur Spaltung einer Rechtsordnung. Dies gilt insbesondere dann, wenn die sprachlichen Differenzen zugleich politische und/oder kulturelle Grenzen markieren. So wird etwa für Belgien seit geraumer Zeit eine zunehmende Spaltung des Rechtssystems in einen wallonischen und einen flämischen Teil beobachtet. Beide Teile nutzen unterschiedliche Referenzwerke und Zeitschriften, sind von unterschiedlichen Denkweisen geprägt, und sogar die Gerichtsentscheidungen sollen je nach dem sprachlichen Hintergrund der Richter verschieden ausfallen. ${ }^{56}$

\section{Regelungseinheit über die mitgliedstaatlichen Grenzen}

Privatrechtliche Normen, die ein bestimmtes Regelungsproblem lösen sollen, haben keineswegs immer einen unterschiedlichen Inhalt in den verschiedenen europäischen Staaten. Vielmehr sind zahlreiche Materien des Privatrechts grenzüberschreitend einheitlich geregelt. Hierfür gibt es eine Reihe von Gründen. Eine lange Tradition hat das Einheitsprivatrecht der internationalen Abkommen (dazu unter 1.). Auch ohne die rechtliche Verbindlichkeit dieser Abkommen kann eine Kooperation zwischen den Gesetzgebern mehrerer Staaten zu einheitlichen Lösungen im Privatrecht führen (dazu unter 2.). Zudem ist die Europäische Union seit geraumer Zeit im Bereich des Privatrechts gesetzgeberisch tätig und schafft mit ihren Mitteln einheitliche privatrechtliche Regelungen (dazu unter 3.). Schließlich kann es selbst ohne jede gesetzgeberische Interventionen zur spontanen Kreation von Einheitsrecht durch Wissenschaft und Praxis kommen (dazu unter 4.).

\section{Internationale Abkommen}

Die klassische Quelle des Einheitsrechts bilden internationale Konventionen. ${ }^{57}$ Als Gegenreaktion auf die Nationalisierung des Rechts und die damit verbundene Regelungsvielfalt, die man bald als Hindernis für den wachsenden grenzüberschreitenden Handel erkannte, wurden seit der zweiten Hälfte des 19. Jahrhunderts zunehmend internationale Übereinkommen abgeschlossen, die für einheitliche Normen in bestimmten Bereichen des Privatrechts sorgen sollten. ${ }^{58}$ Heute existieren derartige Übereinkommen in einer Vielzahl von Sachgebieten von zumeist wirtschaftlicher Bedeutung. Aber auch im Familien- und Erbrecht gibt es eine Reihe von Konventionen,

55 Umfassend hierzu Richter, Sprachenordnung und Minderheitenschutz im schweizerischen Bundesstaat, 2005, passim.

56 Vgl. Heirbaut, in: Bocken/De Bondt (Hrsg.), Introduction to Belgian Law, 2001, S. 1, 16 ff.; siehe aber auch die wesentliche optimistischere Einschätzung der Einheitlichkeit des belgischen Rechts bei Schübel-Pfister, Sprache und Gemeinschaftsrecht, 2004, S. 153 f.

57 Vgl. immer noch grundlegend Kropholler, Internationales Einheitsrecht, 1975, S. 93 ff.

58 Ausführlich dazu Bischoff, Die Europäische Gemeinschaft und die Konventionen des einheitlichen Privatrechts, S. $25 \mathrm{ff}$. 
die etwa für ein einheitliches Kollisionsrecht sorgen sollen..$^{59}$ Der Kernbereich des Privatrechts, d.h. das Vertrags-, Schuld- und Sachenrecht blieb freilich bis heute weitgehend unberührt. Eine praktisch bedeutsame Ausnahme bildet lediglich das UN-Kaufrecht (CISG), das mittlerweile in 77 Staaten in Kraft getreten ist. ${ }^{60}$ Das CISG findet grundsätzlich Anwendung auf Kaufverträge zwischen Parteien, die ihren Sitz in verschiedenen Staaten haben, wenn diese Staaten Vertragsstaaten sind, oder durch das Kollisionsrecht auf das Recht eines Mitgliedsstaates verwiesen wird (Art. 1 Abs. 1 CISG). Ausgenommen sind jedoch insbesondere Kaufverträge über Güter zum privaten Gebrauch (Art. 2 (a) CISG). Da das CISG auch in allen Mitgliedstaaten der Union mit Ausnahme Irlands, Portugals, Maltas und des Vereinigten Königreichs gilt, steht für den nach wie vor zentralen Vertragstyp im grenzüberschreitenden Handel zwischen Unternehmern fast überall in Europa ein einheitliches Recht zur Verfügung.

\section{Kooperation der Gesetzgeber}

Insbesondere in den nordeuropäischen Staaten Dänemark, Finnland, Island, Norwegen und Schweden gibt es eine lange Tradition der gesetzgeberischen Zusammenarbeit. ${ }^{61}$ Diese Zusammenarbeit zeichnet sich durch einen vorwiegend informellen, unbürokratischen und freiwilligen Charakter aus. ${ }^{62}$ Zwar findet sie seit dem 1.7.1962 eine vertragliche Grundlage in einem in Helsinki abgeschlossenen Abkommen. Aber dieses Abkommen enthält keine zwingenden Normen, so daß der Erfolg der Kooperation auch weiterhin von der freiwilligen Übernahme der gemeinsam entwickelten Vorschriften in den einzelnen skandinavischen Staaten abhängt.

Nach einer Blüte der Zusammenarbeit in den 1950er und 60er Jahren wird seitdem eine stetige Abnahme der gemeinsam erzielten Fortschritte beobachtet. ${ }^{63}$ Gleichwohl existiert weiterhin ein aktiver nordischer Rat der Justizminister (MR-LAG), der zudem mit den baltischen Staaten zusammenarbeitet und so für größere Rechtseinheit im Ostseeraum sorgen will. Die gesetzgeberische Zusammenarbeit hat trotz ihres unverbindlichen Charakters zu einer weitgehenden Regelungseinheit im Haftpflichtund Schadensersatzrecht, im Vertragsrecht und sogar im Familien- und Erbrecht geführt, ${ }^{64}$ während eine frühere Einheit im Bereich des Gesellschaftsrechts in jüngerer

59 Ausführlich zu den Sachgebieten der Übereinkommen des einheitlichen Privatrechts Bischoff (Fn. 58), S. $44 \mathrm{ff}$.

60 Vgl. Piltz, NJW 2011, 2261; seitdem ist das CISG am 1.8.2012 auch in Benin in Kraft getreten.

61 Vgl. dazu einführend Carsten, ZEuP 1993, 335, $341 \mathrm{ff} . ;$ Bernitz, Scandinavian Studies in Law 39 (2000), 29, 33 ff.; siehe auch bereits Kropholler (Fn. 57), S. $109 \mathrm{ff}$.

62 So Carsten, ZEuP 1993, 335, 342; Bernitz, Scandinavian Studies in Law 50 (2007), 13, 24.

63 Vgl. Bernitz, Scandinavian Studies in Law 50 (2007), 13, 23 ff.; ders., Scandinavian Studies in Law 39 (2000), 29, $40 \mathrm{ff}$.

64 Vgl. Carsten, ZEuP 1993, 335, $345 \mathrm{ff}$. 
Zeit offenbar teilweise verlorengegangen ist. ${ }^{65}$ Der Grad der Einheitlichkeit muß indes für jede Materie jeweils gesondert bestimmt werden.

Die Einheit des nordischen Rechtsraums wird nicht nur durch die Kooperation der Justizminister gefördert, sondern auch im Rahmen des alle drei Jahre stattfindenden gemeinsamen nordischen Juristentags stets von neuem gestärkt. ${ }^{66}$ Durch den solchermaßen institutionalisierten grenzüberschreitenden Diskurs konnte es zu einer Identifikation der skandinavischen Juristen mit ihrem gemeinsamen Rechtsraum kommen, der in der Rechtsvergleichung auch verbreitet als eigener skandinavischer Rechtskreis anerkannt wird. ${ }^{67}$

Eine grenzüberschreitende Zusammenarbeit, die u.a. ebenfalls auf eine Vereinheitlichung des Zivilrechts abzielte, wurde durch den sogenannten Benelux-Vertrag, der 1960 in Kraft trat, auch zwischen Belgien, den Niederlanden und Luxemburg begründet. ${ }^{68}$ Freilich reichten die Ergebnisse dieses Vereinheitlichungsprojekts nie an diejenigen der skandinavischen Staaten heran, ${ }^{69}$ und infolge der zunehmenden Integration der Benelux-Staaten in der Europäischen Gemeinschaft verlor der BeneluxVertrag noch zusätzlich an praktischer Bedeutung. ${ }^{70}$ Nichtsdestotrotz wurde 2008 eine unbefristete Neufassung des Benelux-Vertrags in Den Haag feierlich unterzeichnet, ${ }^{71}$ der recht ambitionierte politische Ziele formuliert. ${ }^{72}$

\section{Rechtssetzung der Europäischen Union}

Einen besonders wichtigen Beitrag zur Vereinheitlichung des Privatrechts in Europa hat in den letzten Jahrzehnten die Europäische Gemeinschaft bzw. die Europäische Union geleistet. Nach einigen Rechtsakten in zivilrechtlichen Nebengebieten wie vor

65 Vgl. Bernitz, Scandinavian Studies in Law 50 (2007), 13, 25.

66 Zuletzt der 39. Nordische Juristentag vom 18. bis 19. August 2011 in Stockholm. Allgemein zum Nordischen Juristentag und seiner Bedeutung für die Einheit des nordischen Rechts Carsten, RabelsZ 37 (1973), $80 \mathrm{ff}$.

67 Vgl. Zweigert/Kötz, Einführung in die Rechtsvergleichung, 3. Aufl. 1996, S. 270 ff.; Carsten, ZEuP 1993, 335, $339 \mathrm{ff}$.

68 Ausführlich dazu Schmiedel, in: in: Basedow/Hopt/Zimmermann (Hrsg.), Handwörterbuch des europäischen Privatrechts, Bd. I, 2009, S. 177 ff.

69 Diese lagen vor allem im Bereich des Wirtschaftsrechts, vgl. Schmiedel (Fn. 68), S. 180.

70 Vgl. Linhart, Internationales Einheitsrecht und einheitliche Auslegung, 2005, S. $107 \mathrm{f}$.

71 Vertrag zur Neufassung des am 3. Februar 1958 unterzeichneten Vertrags zur Errichtung der BeneluxWirtschaftsunion.

72 Vgl. Schmiedel (Fn. 68), S. 182. 
allem dem Arbeitsrecht ${ }^{73}$ und dem Gesellschaftsrecht ${ }^{74}$ ist der europäische Gesetzgeber seit Mitte der 80er Jahre des letzten Jahrhunderts auch im Bereich des allgemeinen Privatrechts in stetig zunehmendem Maß tätig geworden. ${ }^{75}$ Ein besonderer Schwerpunkt lag dabei auf dem Verbraucherschutzrecht. Insbesondere mit Hilfe des Instruments der Richtlinie versuchte der europäische Gesetzgeber, in diesem Gebiet für einheitliche europäische Standards zu sorgen. Diese Strategie hat sich jedoch als unzweckmäßig erwiesen. Denn die Richtlinie ist grundsätzlich nicht unmittelbar anwendbar, sondern bedarf zunächst der Umsetzung durch den mitgliedstaatlichen Gesetzgeber $^{76}$, der dabei regelmäßig einen gewissen Umsetzungsspielraum besitzt ${ }^{77}$. Daher existieren auch im angeglichenen Recht weiterhin Differenzen zwischen den mitgliedstaatlichen Rechtsordnungen.

Die europäische Kommission sieht in diesen Differenzen seit geraumer Zeit ein Problem für die volle Entfaltung des europäischen Binnenmarkts. Denn Verbraucher und Unternehmer würden durch die bestehende Regelungsvielfalt von grenzüberschreitenden Transaktionen abgeschreckt. Die Kommission entwickelte zwei Lösungsansätze, um dem Problem der mangelnden Einheit des europäischen Privatrechts zu begegnen. Erstens wollte sie vom bisherigen Prinzip der Mindestharmonisierung abgehen und stattdessen eine Vollharmonisierung erreichen, indem sie den Umsetzungsspielraum der Mitgliedstaaten abschaffen bzw. einengen wollte. Dieser Ansatz ist freilich aufgrund politischen Widerstands weitgehend gescheitert. ${ }^{78}$ Zweitens hat die Kommission einen Vorschlag für eine Verordnung über ein Gemeinsames Europäisches Kaufrecht vorgelegt. ${ }^{79}$ Dieses Kaufrecht soll als sogenanntes Optiona-

73 Vgl. zur Gleichbehandlung die Richtlinien 75/117/EWG, 76/207/EWG und 79/7/EWG, zum technischen Arbeitsschutz die Richtlinien 77/578/EWG und 80/107/EWG und zur sozialen Sicherheit der Arbeitnehmer die Richtlinien 75/129/EWG, 77/187/EWG und 80/987/EWG. Siehe zu dieser frühen Entwicklung des Europäischen Arbeitsrechts etwa Thüsing, Europäisches Arbeitsrecht, 2. Aufl. 2008, S. $5 \mathrm{ff}$.

74 Vgl. die Erste Richtlinie v.9.3.1968 (68/151/EWG, Publizitätsrichtlinie), die Zweite Richtlinie v. 13.12.1976 (77/91/EWG, Kapitalrichtlinie), die Dritte Richtlinie v. 9.10.1978 (78/855/EWG, Fusionsrichtlinie), die Vierte Richtlinie v. 25.7.1968 (78/660/EWG, Jahresabschlußrichtlinie), die Sechste Richtlinie v. 17.12.1982 (82/891/EWG, Spaltungsrichtlinie) und die Siebente Richtlinie v. 13.6.1983 (83/349/EWG, Richtlinie über den konsolidierten Abschluß).

75 Vgl. Basedow, AcP 210 (2010), 164 ff.

76 Soweit der Gesetzgeber seiner Aufgabe nicht oder nur unzureichend nachkommt, sind die Gerichte aufgefordert, den Vorgaben der Richtlinie möglichst im Wege der richtlinienkonformen Auslegung des nationalen Rechts Rechnung zu tragen, vgl. nur EuGH, Urt. v. 24.5.2012, C-97/11 (Amia), Slg. 2012, I-0000, Rn.28; EuGH, Urt. v.24.1.2012, C-282/10 (Maribel Dominguez), Slg. 2012, I-0000, Rn. 24 m.w.N.

77 Auch eine richterliche Rechtsangleichung durch eine richtlinienkonforme Auslegung des nationalen Rechts kann in solchen Fällen nicht für Rechtseinheit sorgen, weil bereits die Vorgaben der Richtlinie nicht präzise genug sind und Spielräume für nationale Sonderwege offenlassen.

78 Siehe aber auch die positivere Einschätzung der neuen Verbraucherrechterichtlinie bei Schwab/Giesemann, EuZW 2012, 253 ff.; ausführlich zum Gesetzgebungsverfahren dieser Richtlinie Unger, ZEuP 2012, 270, 273 ff.

79 Vorschlag für eine Verordnung des Europäischen Parlaments und des Rates über ein Gemeinsames Europäisches Kaufrecht, KOM (2011) 635 endgültig. Einführend hierzu etwa Schmidt-Kessel, in: ders. (Hrsg.), Ein einheitliches europäisches Kaufrecht?, 2012, S. 1 ff. 
les Instrument vor allem für grenzüberschreitende Kaufverträge zwischen Verbrauchern und Unternehmern von den Vertragsparteien frei wählbar sein. Mit diesem Optionalen Instrument soll für solche grenzüberschreitenden Kaufverträge ein einheitliches Recht in dem gesamten Raum der Europäischen Union bereitstehen.

Der Erlaß einer Verordnung über ein Gemeinsames Europäisches Kaufrecht allein wird indes keine praktisch wirksame Rechtseinheit in Europa garantieren können. Denn auch in der europäischen Rechtsordnung wirken einige der einheitsgefährdenden Faktoren, die schon innerhalb der mitgliedstaatlichen Rechtsordnungen für regionale Regelungsdifferenzen trotz einheitlich geltenden Gesetzesrechts sorgen. ${ }^{80}$

So stellt insbesondere die Sprachenvielfalt der mittlerweile 23 gleichberechtigten Amtssprachen die Rechtsordnung der Europäischen Union vor gewaltige Probleme, die trotz des betriebenen enormen finanziellen Aufwands bislang nur unzureichend gelöst sind. Den Texten des europäischen Rechts, die in allen Sprachversionen die gleiche Verbindlichkeit besitzen, läßt sich ihr Inhalt durch die bloße Lektüre des Wortlauts regelmäßig kaum mit der Sicherheit entnehmen, die die Rechtsanwender aus ihren mitgliedstaatlichen Rechtsordnungen gewohnt sind.

Praktische Rechtseinheit besteht außerdem regelmäßig nur dann, wenn ein hierarchisch gegliedertes Gerichtssystem mit einem starken Höchstgericht an der Spitze eine einheitliche Rechtsprechung garantiert. Denn Rechtsnormen können stets nur mehr oder weniger weite Rahmenvorgaben machen, die in der Praxis konkretisiert und an die sich wandelnden Umstände angepaßt werden müssen. Soweit diese Rechtsfortbildung unter den Gerichten nicht effektiv koordiniert wird, wird es zu unterschiedlichen Interpretationen des geltenden Rechts kommen. In der Europäischen Union steht mit dem Vorlageverfahren nach Art. 267 AEUV zwar prinzipiell ein Verfahren bereit, das die Auslegung des europäischen Rechts beim EuGH konzentrieren soll. Es ist freilich sehr zweifelhaft, ob dieses Vorlageverfahren und das bestehende europäische Gerichtssystem geeignet sind, mit der Zunahme an Verfahren fertig zu werden, die bei einer erfolgreichen Einführung eines gemeinsamen europäischen Kaufrechts zu erwarten wären. Bereits heute wird eine erhebliche Arbeitsüberlastung des Europäischen Gerichtshofs beklagt. ${ }^{81}$ Zudem verzögert das Vorlageverfahren, das nicht in den normalen Instanzenzug integriert ist, die Zivilprozesse in Europa teilweise erheblich. Eine dezentrale Koordination derart, dass die nationalen Gerichte ihre Entscheidungen spontan anglichen, kommt als wirksame Alternative wenigstens derzeit nicht in Betracht. Zwar ist anzuerkennen, dass die europäischen Gerichte vor allem bei Auslegungsfragen hinsichtlich des Unionsrechts

80 Vgl. zum folgenden auch bereits Max Planck Institute for Comparative and International Private Law, RabelsZ 75 (2011), 371, $431 \mathrm{ff}$.

81 Siehe dazu jüngst Klinke, EuR - Beiheft 1 - 2012, 61, 63 ff. mwN. 
Rechtsprechung aus anderen Mitgliedstaaten stärker als früher beachten ${ }^{82}$. Doch stellen solche rechtsvergleichenden Analysen in der höchstrichterlichen Rechtsprechung angesichts der praktischen Schwierigkeiten solcher Arbeit immer noch seltene Ausnahmen dar. Zudem besteht keine strenge Bindung an die Entscheidungen fremder Rechtsordnungen, so dass sich verlässliche Rechtseinheit auf diesem Wege kaum herstellen lässt. Ein Gemeinsames Europäisches Kaufrecht sollte deshalb nur dann eingeführt werden, wenn zugleich das europäische Gerichtssystem grundlegend reformiert wird. ${ }^{83}$

\section{Spontane Rechtsangleichung durch Wissenschaft und Praxis}

Die Angleichung von Rechtsordnungen bedarf nicht zwingend staatlicher Interventionen, sondern kann auch spontan durch Wissenschaft und Praxis herbeigeführt werden. Obwohl es bis heute an einem europäischen juristischen Diskurs fehlt, wie er zu Zeiten des ius commune bereits einmal bestand, ${ }^{84}$ hat das Gespräch über die mitgliedstaatlichen Grenzen hinweg in den letzten Jahrzehnten erheblich zugenommen. Zahlreiche internationale Zeitschriften sind erschienen, in denen juristische Themen von europäischer Bedeutung behandelt werden. Zudem haben viele Forschergruppen nach dem Vorbild der sogenannten Principles of European Contract Law $^{85}$ auf der Grundlage der mitgliedstaatlichen Rechtsordnungen Modellregelwerke für die verschiedensten Privatrechtsgebiete entwickelt. ${ }^{86}$ Die europäische Privatrechtsvergleichung hat dadurch in jüngerer Zeit einen erheblichen Aufschwung er-

82 Vgl. etwa für Deutschland BGH, Urt. v. 22.1.2009, I ZR 125/07, JZ 2009, 856 ff.; BGH, Urt, v. 22.4.2009, VIII ZR 156/07, NJW 2009, 2606 ff.; für England Kirin-Amgen v Transkaryotic Therapies [2004] UKHL 46 at para. 73 ff. per Lord Hoffmann mit der bemerkenswerten Aussage, dass „the distinguished German patent lawyer Dr Rüdiger Rogge (then presiding judge of the 10th (intellectual property) Senate of the Bundesgerichtshof) said that he regarded the decisions of other countries on the extent of protection afforded by Art, 69 [European Patent Convention] as important contributions to the jurisprudence of his own country. The same is true of the judges of the United Kingdom".

83 Für die Einführung einer Revision zum Europäischen Gerichtshof etwa Bruns, JZ 2011, $325 \mathrm{ff}$.

84 Vgl. nur Coing, Europäisches Privatrecht, Bd. I, 1985, S. 7 ff., 39 ff.

85 Lando/Beale, Principles of European Contract Law, Parts I and II, 1999; Lando/Clive/Prüm/Zimmermann, Principles of European Contract Law, Part III, 2003; zu den PECL und der europäischen Rechtswissenschaft siehe Zimmermann, Die Principles of European Contract Law als Ausdruck und Gegenstand europäischer Rechtswissenschaft, 2004.

86 Neben den PECL wurden bislang folgende vergleichbare Principles veröffentlicht: Hayton/Kortmann/Verhagen (Hrsg.), Principles of European Trust Law, 1999; McBryde/Flessner/Kortmann (Hrsg.), Principles of European Insolvency Law, 2003; European Group on Tort Law (Hrsg.), Principles of European Tort Law, 2005; Boele-Woelki (Hrsg. u.a.), Principles of European Family Law Regarding Divorce and Maintenance Between Former Spouses, 2004; dies. (Hrsg. u.a.), Principles of European Family Law Regarding Parental Responsibilities, 2007; Basedow/Birds/Clarke/Cousy/Heiss (Hrsg.), Principles of European Insurance Contract Law, 2009. Des weiteren gibt es Ideen zur Entwicklung von ähnlichen Principles im Bereich des Geistigen Eigentums, vgl. Obly, ZGE/IPJ 2 (2010), 365 ff., und im Arbeitsrecht, vgl. Waas, International Journal of Comparative Labour Law and Industrial Relations 24 (2008), 451, 454 ff. Einen Überblick über die 2003 schon existierenden verschiedenen Arbeitsgruppen bietet Wurmnest, ZEuP 2003, $714 \mathrm{ff}$. 
fahren, und die Zeiten einer rein nationalen „Landesjurisprudenz“, wie sie einst von Jhering zurecht beklagt wurden, ${ }^{87}$ scheinen überwunden zu sein.

Allerdings beschränkt sich die rechtsvergleichende Arbeit bislang noch weitgehend auf die Wissenschaft und spielt in der täglichen Arbeit der Gerichte in Europa eine eher untergeordnete Rolle. ${ }^{88}$ Doch auch hier ist Bewegung zu erkennen. So hat der ehemalige Präsident des Bundesgerichtshofs Walter Odersky bereits 1994 für eine harmonisierende Auslegung des nationalen Rechts durch die Gerichte plädiert. ${ }^{89}$ Seitdem sind einige Netzwerke europäischer Gerichte geknüpft worden, ${ }^{90}$ und der Austausch der Richter über die Grenzen hinweg hat sich intensiviert, so daß auch hier die nationale Isolation langsam aufgebrochen wird. Rechtsvergleichende Betrachtungen stellen die Gerichte vor allem in gesellschaftlich umstrittenen und moralisch schwierigen Fragen wie etwa den sogenannten „wrongful life“-Fällen an ${ }^{91}$, oder wenn das eigene Recht bei komplizierten Problemen keine Lösung bietet, sich eine solche aber in einer fremden Rechtsordnung findet ${ }^{92}$. Und nicht zuletzt nimmt die Rechtsprechung häufig eine aktive Rolle bei der effektiven Umsetzung der Vorgaben des Unionsrechts ein, wenn sie das nationale Recht richtlinienkonform auslegt ${ }^{93}$.

Einen kaum zu überschätzenden und bislang nur unzureichend untersuchten Beitrag zur Vereinheitlichung des Wirtschaftsrechts hat die internationale Kautelarpraxis geleistet. Global agierende Großkanzleien gebrauchen heute überall grundsätzlich möglichst einheitliche Vertragsmuster, und internationale Organisationen geben Standardklauseln heraus, die weltweit Verwendung finden. Von besonderer Bedeutung sind hier die sogenannten Internationalen Handelsklauseln (Incoterms) und die

87 Vgl. Jhering, Geist des römischen Rechts, Erster Theil, 5. Aufl. 1891, S. 15, mit der Forderung nach neuer Universalität im Sinne einer vergleichenden Jurisprudenz.

88 Vgl. für eine differenzierte Darstellung die Länderberichte in Drobnig/van Erp (Hrsg.), The Use of Comparative Law by Courts, 1999, S. 25 ff. Rechtsvergleichende Untersuchungen werden häufiger von Gerichten kleinerer Staaten angestellt, die sich an der Rechtsprechung benachbarter Staaten orientieren, vgl. etwa Elvinger, in: Drobnig/van Erp (Hrsg.), The Use of Comparative Law by Courts, S. 231, 234.

89 Odersky, ZEuP 1994, 1 ff.

90 So etwa das Network of the Presidents of the Supreme Judicial Courts of the European Union (http:// www.network-presidents.eu/), die Association of the Councils of State and Supreme Administrative Jurisdictions of the European Union (http://www.juradmin.eu/), das European Judicial Training Network (EJTN, http://www.ejtn.net/) und die Association des Hautes Jurisdictions de Cassation des Pays ayant en partage l'Usage du Français (AHJUCAF, http://www.ahjucaf.org/).

91 Vgl. hierzu die Nachweise bei Giesen, Utrecht Law Review 8 (2012), 35 ff.; für Deutschland siehe beispielsweise auch BGHZ 176, 365, 374 zur rechtlichen Beurteilung einer Scheidung mosaischen Rechts mit rechtsvergleichenden Nachweisen zur Rechtsprechung der Cour de cassation und des kanadischen Supreme Court.

92 Vgl. etwa die Entscheidung des englischen House of Lords in Fairchild v Glenhaven [2003] 1 AC 32, 117 per Lord Rodger of Earlsferry.

93 Vgl. nur die „Quelle“-Entscheidung des BGH (BGHZ 179, 27 ff.), in welcher der BGH eine richtlinienkonforme Auslegung vornahm, die er in einer früheren Entscheidung noch als contra legem abgelehnt hatte. Dazu etwa Höpfner, EuZW 2009, 159 f.; Schinkels, JZ 2011, 394 ff. 
Einheitlichen Richtlinien und Gebräuche für Dokumenten-Akkreditive (ERA) der Internationalen Handelskammer (International Chamber of Commerce, ICC) ${ }^{94}$ Solches privat gesetztes Recht ist zu seiner Durchsetzung zwar stets auf eine staatliche Anerkennung angewiesen und leitet seine Geltung daher formal immer von einer staatlichen Rechtsordnung ab. ${ }^{95}$ Aber im Rahmen der Dispositionsfreiheit, die gerade im Handelsrecht besonders groß ist, kann durch privatautonome Gestaltungen in weitem Umfang ein eigenes materielles Recht entstehen, das für den Rechtsalltag sogar von größerer Bedeutung als das staatlich gesetzte Recht ist.

\section{E. Ergebnis}

Die Formel „Ein Staat = eine (eigene) privatrechtliche Regelung “ hat sich als überaus ungenau erwiesen. Das europäische Privatrecht läßt sich nicht in die nationalen Grenzen einzwängen, sondern weist eine komplexe Regelungsvielfalt auf. Dabei finden sich etwa in Schottland und, wenn auch in geringerem Umfang, in Katalonien, so umfangreiche eigenständige Regelungsstrukturen, daß man sogar von selbständigen Privatrechtsordnungen sprechen kann. Aber auch sonst gibt es in vielen Bereichen innerhalb der Mitgliedstaaten der Europäischen Union regional unterschiedliche privatrechtliche Regelungen. Diese Unterschiede lassen sich auf eine Reihe von Gründen zurückführen.

So gibt es regionale Sondergesetze oder Zentralgesetze, die nur für bestimmte Regionen gelten. Solche gesetzlichen Sonderregelungen betreffen zwar regelmäßig nicht den traditionellen Kern des Privatrechts, d.h. das Vertrags- und Schuldrecht. Aber sie sind doch häufig im Erbrecht und bestimmten Bereichen des Liegenschaftsrechts anzutreffen, wo historische Besonderheiten fortgeschrieben werden. Historische Gründe können gelegentlich auch in anderen Spezialgebieten fortbestehende gesetzliche Sonderregelungen erklären, so etwa spezielle Bestimmungen im Insolvenzrecht für das Elsaß. Zudem kann regionales Gewohnheitsrecht entstehen, oder eine Geschäftspraxis lokal begrenzt sein. In einem Staat können sich auch unterschiedliche Rechtsprechungslinien herausbilden, wenn und soweit die Instanzgerichte nicht der Kontrolle durch ein Höchstgericht unterliegen. Und schließlich können sprachliche Besonderheiten rechtliche Differenzierungen nach sich ziehen.

Abweichungen von der Formel „Ein Staat = eine (eigene) privatrechtliche Regelung “ können sich des Weiteren aus der Geltung von Normen mit grenzüberschreitend einheitlichem Inhalt ergeben. Solch einheitliches Recht kann auf internationalen Abkommen oder einer sonstigen allgemeinen Kooperation verschiedener nationaler Gesetzgeber beruhen. In jüngerer Zeit hat vor allem die Europäische Union die Ver-

94 Vgl. dazu nur Buck-Heeb/Dieckmann, Selbstregulierung im Privatrecht, 2010, S. 148 ff.

95 So etwa der Einwand von Ipsen, Private Normenordnung als Transnationales Recht?, 2009, S. 82. 
einheitlichung des europäischen Privatrechts vorangetrieben. Wichtige Beiträge zur spontanen Angleichung leisten zudem Wissenschaft und Praxis.

Die regionale Reichweite einer privatrechtlichen Norm in Europa läßt sich also nicht an einer einfachen Formel ablesen, sondern muß stets im Einzelfall bestimmt werden. Der traditionelle Anspruch der europäischen Staaten auf Regelungssouveränität und Rechtseinheit in ihrem gesamten Staatsgebiet konnte nie ganz durchgesetzt werden und ist heute in weiten Bereichen des Privatrechts stark relativiert. 